\title{
ORIGINAL
}

\section{EL PERFIL DE LA DEPENDENCIA EN ESPAÑA: UN ANÁLISIS DE LA ENCUESTA DE DISCAPACIDADES DE 1999 (*)}

\author{
Rosa Gispert Magarolas (1), Guillem Clot-Razquin (1), Antoni Rivero Fernández (2), Adriana \\ Freitas Ramírez (1), Miguel Ruíz-Ramos (3), Cecilia Ruíz Luque (4), Ester Busquets Bou (5), \\ Josep $\mathrm{M}^{\mathrm{a}}$ Argimón Pallàs (6).
}

(1) Servei d'Informació i Estudis. Departament de Salut. Generalitat de Catalunya.

(2) Fundació Institut Català de l’Envelliment. Universitat Autònoma de Barcelona. Barcelona.

(3) Instituto Andaluz de Estadística. Junta de Andalucía. Sevilla.

(4) Institut d'Estadística de Catalunya. Generalitat de Catalunya. Barcelona.

(5) Institut Català de la Salut. Generalitat de Catalunya. Barcelona.

(6) Servei Català de la Salut. Generalitat de Catalunya. Barcelona.

\section{RESUMEN}

Fundamentos: En los próximos años se producirá un importante envejecimiento demográfico en España y con ello habrá un mayor nivel de comorbilidad, discapacidad y dependencia. El objetivo del trabajo es estimar el perfil de la dependencia , a partir del nivel de gravedad de las diferentes discapacidades padecidas por la población española.

Métodos: Se utilizó la Encuesta sobre Discapacidades, Deficiencias y Estado de Salud de 1999 del INE, que incluye 36 discapacidades específicas y su gravedad (de 0:ausencia de discapacidad a 4: no puede realizar). Se agruparon 5 tipos de discapacidad, se calculó la gravedad media de cada tipo y se agregó en 5 grados de dependencia. Para relacionar cada discapacidad específica con la gravedad se realizó un análisis de correspondencias simples. Para identificar los perfiles de dependencia, se realizó un análisis de correspondencias múltiples y uno de clusters teniendo en cuenta la edad, el sexo, tipos de discapacidad y grado de dependencia.

Resultados: Se observó asociación entre la gravedad y las discapacidades específicas (mayor gravedad en los que sufren limitaciones en las actividades de la vida diaria). La dependencia se caracterizó en cinco grupos: los de menor dependencia son preferentemente de edades medias, varones y con discapacidades sensoriales y entre las personas con dependencia más elevada predominan mujeres, mayores de 80 años y las discapacidades de movilidad y en las actividades de la vida diaria.

Conclusiones: Se identifican cinco grupos de personas dependientes y este estudio permite estimar su frecuencia global dentro de la población, lo que puede ser de gran utilidad para estimar los recursos necesarios para su atención.

Palabras clave: Discapacidad y dependencia. Actividades de la vida diaria. Envejecimiento. Cluster análisis.

Correspondencia:

Rosa Gispert

Servei d'Informació i Estudis. Departament de Salut

C/ Travessera de les Corts, 131-159

Pavelló Ave Maria. 08028 Barcelona

correo electrónico: rosa.gispert@gencat.cat

\section{ABSTRACT}

\section{Dependence profile in Spain: An analysis from the disability survey of 1999}

Background: In the next future an important demographical ageing will imply a rise in the levels of co morbidity, disability and dependence of the population. The objective if this work was to estimate the dependence's profile by means of assessing the levels of severity of the disability in the Spanish general population.

Methods: Data come from the disease, disability and health status survey of 1999 of the Spanish Institute for Statistics, which includes 36 limitations with an score of severity (from $0=$ absence to $4=$ unable). These limitations were grouped in five kinds of disability, the mean score of severity was computed for each one, and grouped in five levels of dependence. To check the relationship between limitations and severity an analysis of correspondence was performed. To identify the pattern of dependence a multiple correspondence and a clusters analysis were computed using the variables of age, sex, kind of disability and level of dependence.

Results: There is a relationship between severity and some limitations (being the limitation on daily activities associated to the highest severity). Five groups of dependent people were identified. Those with lower dependence were middle aged, men and having sensorial disabilities and those with higher dependence were mainly women, older than 80 and with disabilities on mobility and on the activities of daily living.

Conclusions: There are five groups of dependent population and this study allows estimating its frequency among the general population what will be very useful to evaluate the amount of resources necessary to take care of them.

Key words: Disabled persons. Activities of daily living. Aging. Clusters analysis.

(*) Financiación proyecto: FIS, PI052650

No se presentan conflictos de intereses. 


\section{INTRODUCCIÓN}

La población de los países occidentales está envejeciendo progresivamente, entre otros motivos por una disminución relativa e importante de la mortalidad en las edades más elevadas ${ }^{1}$. El envejecimiento de la población es uno de los factores que tienen un peso más determinante en la configuración de su nivel de discapaci$\mathrm{dad}^{2}$. Con el aumento de la supervivencia, se acentúa la prevalencia de trastornos crónicos y se incrementa la probabilidad de sufrir discapacidades como consecuencia de la enfermedad ${ }^{3-4}$. Aunque la mayor supervivencia representa un éxito del bienestar de la sociedad, no deja de tener repercusiones importantes en lo que concierne al estado de salud de la población en su conjunto y en la necesidad de servicios sanitarios ${ }^{5-6}$.

La gravedad de esta discapacidad y el impacto para las personas que la sufren en el desarrollo de sus actividades cotidianas determinará su nivel de dependencia, tanto individual como familiar y social ${ }^{7-9}$. El concepto de dependencia fue definido por el Consejo de Europa ${ }^{10}$ como el estado en el que las personas, por razones de falta o pérdida de autonomía física, psicológica o intelectual, requieren asistencia o ayuda significativa para realizar las actividades de la vida diaria. La persona dependiente ha sido definida en el Libro Blanco de la Dependencia ${ }^{11}$ como aquélla que no puede realizar, sin ayuda, alguna de las actividades básicas de la vida diaria relacionadas con el cuidado personal, la movilidad dentro del hogar o las funciones mentales básicas. En los próximos años la proporción de personas mayores y muy mayores probablemente aumentará ocasionando un importante sobreenvejecimiento demográfico en este país, y aunque posiblemente el nivel de personas dependientes no aumente de forma proporcional al envejecimiento, debido al retraso en el inicio de las limitaciones y sus consecuen- cias $^{12}$, habrá un mayor nivel de co-morbilidad y en consecuencia de dependencia ${ }^{3}$. Puesto que la dependencia de las personas puede comportar una importante sobrecarga emocional, física y económica de quienes la sufren y de sus familias (hoy por hoy el soporte asistencial más importante, fundamentalmente a cargo de las mujeres) ${ }^{13}$, conocer la frecuencia y distribución de la discapacidad y la dependencia en la población es fundamental para estimar la necesidad de servicios sanitarios y sociales. La promulgación reciente de la ley que ha de permitir definir las necesidades de ayudas y regular la obtención de los recursos necesarios, confiere además a este tema una especial actualidad sociopolítica ${ }^{14}$.

El objetivo del presente trabajo es estimar el perfil de la dependencia en España, a partir del nivel de gravedad de las diferentes discapacidades padecidas por la población.

\section{MATERIAL Y MÉTODOS}

Los datos proceden de la Encuesta sobre Discapacidades, Deficiencias y Estado de Salud de $1999^{15}$. Esta encuesta se administró a una muestra representativa de hogares y dentro de ellos a las personas no institucionalizadas que presentaban alguna discapacidad. El cuestionario incluye una lista de 36 actividades/funciones que sirve para medir la presencia de discapacidad -nombradas en adelante discapacidades específicas- (tabla 1). Además valora el nivel de dificultad para el desempeño de cada una de estas 36 actividades ("puntuación de severidad" en la encuesta original), de la forma: 0 :no discapacidad, 1:sin dificultad, 2:dificultad moderada, 3:dificultad grave, 4: no puede realizar.

Para el análisis de la gravedad de la discapacidad y del perfil de la dependencia, los datos se agruparon según se muestra en la 
Tabla 1

Discapacidades específicas agrupadas

\begin{tabular}{|c|c|c|c|}
\hline & & $\begin{array}{c}\text { Código de } \\
\text { discapacidad }\end{array}$ & \\
\hline \multirow{7}{*}{ 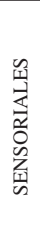 } & \multirow{4}{*}{ VER } & 11 & Percibir cualquier imagen \\
\hline & & 12 & Tareas visuales de conjunto \\
\hline & & 13 & Tareas visuales de detalle \\
\hline & & 14 & Otras discapacidades de visión \\
\hline & \multirow{3}{*}{ OIR } & 21 & Recibir cualquier sonido \\
\hline & & 22 & Audición de sonidos fuertes \\
\hline & & 23 & Escuchar el habla \\
\hline \multirow{11}{*}{ 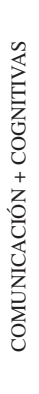 } & \multirow{4}{*}{ COMUNICARSE } & 31 & Comunicarse a través del habla \\
\hline & & 32 & Comunicarse a través de lenguajes alternativos \\
\hline & & 33 & Comunicarse a través de gestos no signados \\
\hline & & 34 & Comunicarse a través de escritura-lectura convencional \\
\hline & \multirow{4}{*}{$\begin{array}{c}\text { APRENDER, } \\
\text { APLICAR CONOCIM. } \\
\text { Y DESARR TAREAS }\end{array}$} & 41 & Reconocer personas, objetos y orientarse \\
\hline & & 42 & Recordar informaciones y episodios \\
\hline & & 43 & Entender y ejecutar órdenes y/o tareas sencillas \\
\hline & & 44 & Entender y ejecutar órdenes y/o tareas complejas \\
\hline & \multirow{3}{*}{$\begin{array}{l}\text { RELACIONARSE CON } \\
\text { OTRAS PERSONAS }\end{array}$} & 101 & Mantener relaciones de cariño con familiares \\
\hline & & 102 & Hacer nuevos amigos y mantener la amistad \\
\hline & & 103 & Relacionarse con compañeros, jefes y subordinados \\
\hline \multirow{9}{*}{ 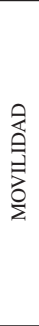 } & \multirow{3}{*}{ DESPLAZARSE } & 51 & Cambiar y mantener las posiciones del cuerpo \\
\hline & & 52 & Levantarse, acostarse, permanecer de pie o sentado \\
\hline & & 53 & Desplazarse dentro del hogar \\
\hline & \multirow{3}{*}{$\begin{array}{c}\text { UTILIZAR BRAZOS } \\
\text { Y MANOS }\end{array}$} & 61 & Trasladar objetos no muy pesados \\
\hline & & 62 & Utilizar utensilios y herramientas \\
\hline & & 63 & Manipular objetos pequeños con manos y dedos \\
\hline & \multirow{3}{*}{$\begin{array}{c}\text { DESPLAZARSE FUERA } \\
\text { DEL HOGAR }\end{array}$} & 71 & Deambular sin medio de transporte \\
\hline & & 72 & Desplazarse en transportes públicos \\
\hline & & 73 & Conducir vehículo propio \\
\hline \multirow{4}{*}{$\sum_{2}^{2}$} & \multirow{4}{*}{$\begin{array}{l}\text { CUIDAR DE } \\
\text { SÍ MISMO }\end{array}$} & 81 & Asearse solo: lavarse y cuidar su aspecto \\
\hline & & 82 & Control de las necesidades y utilizar solo el servicio \\
\hline & & 83 & Vestirse, Desvestirse y arreglarse \\
\hline & & 84 & Comer y beber \\
\hline \multirow{5}{*}{$\sum_{4}$} & \multirow{5}{*}{$\begin{array}{c}\text { REALIZAR LAS } \\
\text { TAREAS DEL HOGAR }\end{array}$} & 91 & Compras y control de los suministros y servicios \\
\hline & & 92 & Cuidarse de las comidas \\
\hline & & 93 & Limpieza y cuidado de la ropa \\
\hline & & 94 & Limpieza y mantenimiento de la casa \\
\hline & & 95 & Cuidarse del bienestar del resto de la familia \\
\hline
\end{tabular}

tabla 1 y se trataron de acuerdo al procedimiento siguiente:

Tipos de discapacidad: las 36 discapacidades específicas se agruparon según el criterio anatómico-funcional usado en otros trabajos $^{15,16}$ en 5 tipos (Di; i=1,2,..,5): sensoriales, cognitivas+comunicación, movilidad, actividades de la vida diaria (AVD), actividades instrumentales de la vida diaria (AIVD), (tabla 1). Cada tipo de discapacidad (Di) incluye un número de discapacidades específicas $\left(\mathrm{dj} ; \mathrm{j}=1, \ldots, \mathrm{k}\right.$ donde $\mathrm{k}=\mathrm{n}^{\mathrm{o}}$ total de discapacidades específicas en cada Di).

Gravedad de la discapacidad: Cada discapacidad específica se acompaña de 
otra variable que recoge una puntuación de "severidad" (Sdj; valores entre 0: no discapacidad y 4: no puede realizar). Para cada "tipo de discapacidad" (Di) se calculó una puntuación media de gravedad (como media de los valores de "severidad" del conjunto de discapacidades específicas de cada tipo) de la siguiente forma:

$$
G D_{i}=\frac{\sum_{j=1}^{k} S d_{j}}{k}
$$

Las discapacidades "percibir cualquier imagen (ceguera)" y "percibir cualquier sonido (sordera)" (con puntuación de severidad 4) y la discapacidad "comer y beber" del grupo de las AVD (con puntuación de severidad ausente en todos los casos) se excluyeron o se analizaron por separado según el caso.

Dependencia: Teniendo en cuenta la existencia o no de discapacidad y la puntuación media de gravedad de cada tipo de discapacidades, se conformaron cinco grados de dependencia para cada uno de los tipos de discapacidad. Las categorías resultantes de dependencia fueron: 0: sin discapacidad, $>0-0,99999$ "discapacidad sin dependencia", 1-1,99999: "dependencia moderada", 2-2,99999: "dependencia grave" y 3-4: "dependencia total".

Se realizó un análisis de correspondencias simples para relacionar cada una de las discapacidades específicas con los niveles de gravedad, excluyendo "ceguera" y "sordera" (del grupo sensoriales) y "comer y beber" (del grupo AVD). Para identificar los perfiles de dependencia entre la población discapacitada, se realizó un análisis de correspondencias múltiples y uno de clusters teniendo en cuenta la edad, el sexo, tipos de discapacidad y grado de dependencia. En este análisis la ceguera y sordera se consideraron en un grupo separado (resultando seis tipos de discapacidad) y se usó la dependencia para cada uno de estos tipos (en 5 categorías). Además, se incluyó la edad (5 categorías) y el sexo (2 categorías) que se trataron como variables suplementarias.

Se utilizó el método de Ward ${ }^{17}$ para realizar la clasificación jerárquica en grupos y se comparó la eficiencia de la clasificación en cuanto a las diferencias entre la inercia y la estabilidad de la agrupación en 5 o en 6 clases. Finalmente se seleccionó la agrupación en cinco clases. Para el análisis se uso el programa estadístico SPAD (Système Portable pour l'Analyse des Données) versión $5.6^{18}$.

\section{RESULTADOS}

En la figura 1 se presentan los resultados gráficos del análisis de correspondencias simple. En ella se muestra la situación en el plano de las discapacidades específicas (3 fueron excluidas) y los niveles de dificultad en su desempeño tal como estaban puntuadas según "severidad" en la encuesta (ver etiquetas de las variables en tabla 1). El análisis pone en evidencia la distancia o proximidad existente entre las distintas discapacidades y la gravedad (y de las distintas discapacidades entre si, de acuerdo con su nivel de gravedad). Los dos factores representados en el gráfico son los que mejor explican los valores observados, pero no los únicos, de forma que hay que tenerlo en cuenta al interpretar la representación gráfica. El factor 1, (eje X) separa claramente el nivel de gravedad "no se puede realizar" de los restantes y el factor 2 (eje Y) la gravedad dentro de las actividades que se pueden realizar (positivo: leve o moderada y negativo: grave). El valor "blanco" representa los valores ausentes de severidad (no informados), que al estar cercanos al punto cero del eje indica que están distribuidos aleatoriamente entre las distintas discapacidades. 
Figura 1

Agrupación de las discapacidades detalladas según gravedad*

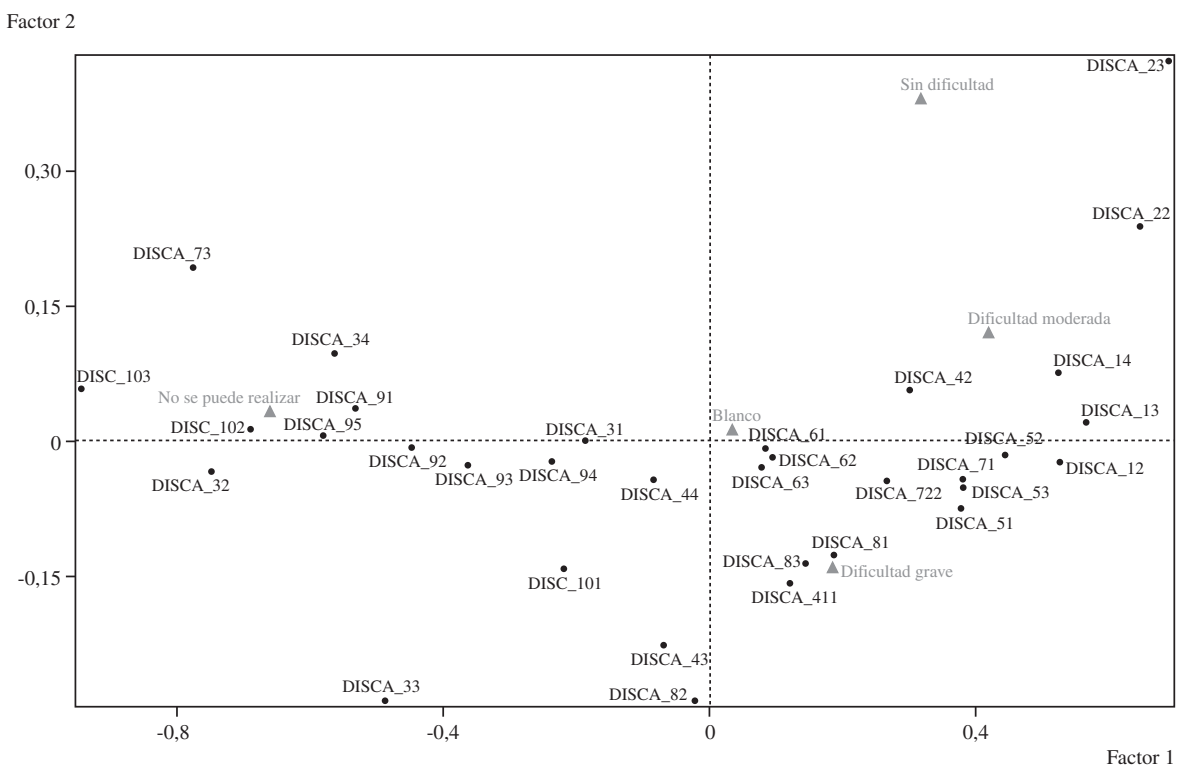

Excluye: 11 percibir cualquier imagen, 21 percibir cualquier sonido, 84 comer y beber. Ver resto de etiquetas de las variables de discapacidad en la tabla 1.

En la interpretación de la figura además hay que tener presente que la distancia representada entre discapacidades y gravedad no es, en sí misma, indicativa de la magnitud de la asociación, sino relativa con respecto al resto de discapacidades. Así por ejemplo, la audición de sonidos fuertes y escuchar el habla (22 y 23) están asociadas a "sin dificultad" y que asearse solo, vestirse-desvestirse y reconocer personas-objetos (discapacidades 81,83 y 41 respectivamente) se asocian a "dificultad grave", más que el resto de discapacidades. Esta figura pone de manifiesto que hay asociación entre la gravedad y el tipo de discapacidad. Así las discapacidades asociadas a las actividades de la vida cotidiana y las instrumentales se sitúan más en la zona de la dificultad grave y total, en cambio las sensoriales y algunas de movilidad en la zona de dificultad moderada. También puede observarse la relación entre las distintas discapacidades de acuerdo a su nivel de gravedad: las discapacidades cognitivas están cerca de las instrumentales, las discapacidades por limitaciones funcionales de los miembros están próximas a las actividades básicas de la vida diaria y las de los problemas de visión se sitúan cercanas a las de movilidad.

En la figura 2 se presenta la distribución de la gravedad según la puntuación media obtenida en cada grupo de discapacidad. Al igual que en el punto anterior no se incluyen las variables ceguera y sordera total que tienen los valores máximos de gravedad. Como puede apreciarse, en general son más frecuentes los niveles de dependencia bajos, con puntuaciones medias entre 0 y 1 ( 0 significa ausencia de ese tipo de discapacidad), con la excepción de las discapacidades en las actividades de la vida diaria AVD y AIVD, en las que se aprecia una frecuencia mayor de valores 2 y 3 en la primera y 4 en la segunda. 
Figura 2

Puntuaciones medias de gravedad según tipo de discapacidad
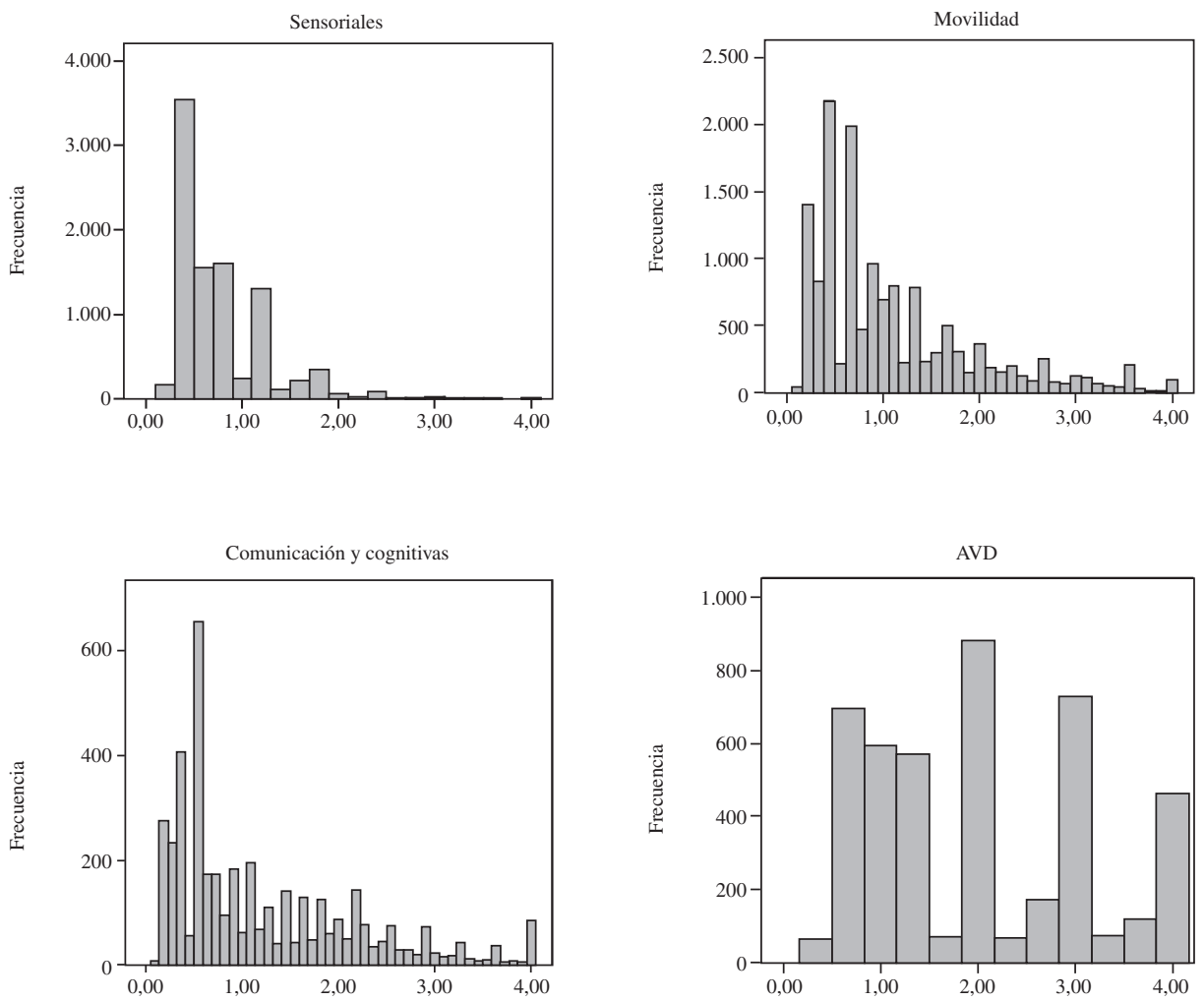

\begin{tabular}{|l|c|c|c|}
\hline Grupo discapacidad & Media & DT & N \\
\hline Sensoriales & 0,76 & 0 & 9.244 \\
\hline Comunicación & 1,22 & 0 & 4.233 \\
\hline Movilidad & 1,10 & 0 & 14.353 \\
\hline AVD & 2,01 & 0 & 4.500 \\
\hline AIVD & 2,17 & 1 & 9.022 \\
\hline
\end{tabular}

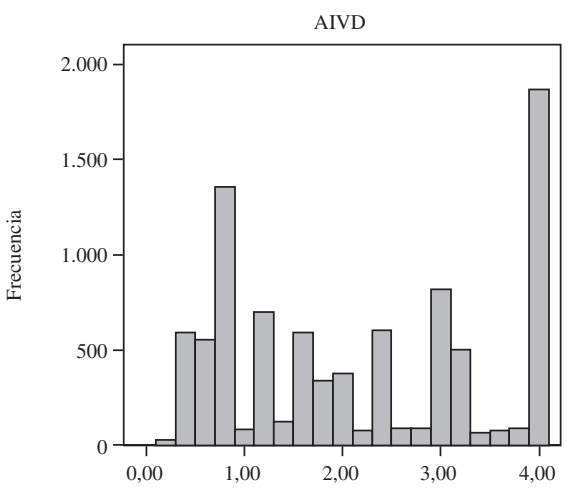

DT: desviación típica.

$\mathrm{N}$ : número de casos. 
En el análisis de correspondencias múltiples (utilizando los tipos de discapacidad con las 5 categorías de dependencia, además de la edad y sexo como complementarias), el histograma de valores propios (no mostrado) indicaba que los tres primeros ejes contenían las relaciones importantes, pero solo explicaban el $28,78 \%$ de la varianza. En el análisis de clusters, dado que el interés del análisis era obtener grupos diferentes entre sí (maximizar la inercia entre clases) y que la partición en 6 clases era menos estable (hay un mayor número de individuos que no quedaban bien clasificados), se seleccionaron 5 clases (tabla 2).

En la tabla 3 se presenta el perfil de la dependencia. Para cada variable (6 tipos de discapacidad con 5 categorías de dependencia, edad y sexo) se muestra la categoría que caracteriza la clase (el grupo de población dependiente). Las diferentes categorías de dependencia en un mismo tipo de discapacidad son auto-excluyentes (por ejemplo, una misma persona no puede tener dependencia moderada y total en sensoriales). Para cada clase se indica la proporción de población discapacitada que incluye.

La primera columna indica el $\%$ de personas de aquella clase que tienen aquella categoría (\% del grupo), por ejemplo el 50,81\% de la clase II son hombres. La segunda columna muestra el $\%$ de las personas que tienen la categoría que se encuentran dentro de la clase $(\%$ de categoría), así el 61,88\% de las personas con dependencia total en las AIVD están en la clase IV y el $21,16 \%$ en la clase $\mathrm{V}$.

El perfil de la dependencia identificado se basa en cinco clases. La primera clase está caracterizada por personas con discapacidades de movilidad pero sin dependencia, varones y de edades entre 40 y 79 años, que agrupa al $34,47 \%$ de los discapacitados. La segunda clase la componen discapacidades sensoriales con poca dependencia, varones y personas de entre 65-79 años, incluyendo al $23,47 \%$ de la población discapacitada. La tercera clase incluye personas con discapacidades de todo tipo pero con dependencias media-bajas. En este grupo hay personas sin discapacidades sensoriales, aunque también incluye ciegos y sordos (que suponen solo el 11,64\% del grupo, pero el $57,6 \%$ de todos los ciegos y sordos). Además está asociada al sexo femenino y edades entre 6 y 64 años, incluyendo al 20,94\% del total de personas con discapacidad. La cuarta clase está formada por personas con un grado de dependencia alto en casi todas las discapacidades excepto las sensoriales. Está asociada a mujeres y personas mayores de 80 años y recoge al $17,18 \%$ de la población con discapacidad. La clase quinta incluye población con un nivel muy alto de dependencia, asociado a mujeres y mayores de 80 años y concentra una parte de las personas que padecen sordera o ceguera. Es el grupo más pequeño suponiendo tan solo el $3,76 \%$ de los discapacitados.

Tabla 2

Análisis de correspondencias múltiples: Inercia entre clases y clasificación de los individuos según número de clases

\begin{tabular}{|c|c|c|c|c|c|}
\hline \multirow{2}{*}{ Clases } & \multirow{2}{*}{ \% Inercia entre clases } & \multicolumn{2}{|c|}{ Individuos } & \multicolumn{2}{c|}{ Individuos } \\
\cline { 3 - 6 } & & Antes & Después & Antes & Después \\
\hline $\mathbf{1}$ & & 4639 & 4747 & 4639 & 4746 \\
\hline 2 & 0,3346 & 7032 & 7003 & 7032 & 6908 \\
\hline 3 & 0,5408 & 4591 & 4234 & 4591 & 4095 \\
\hline 4 & 0,7367 & 3282 & 3475 & 1073 & 1039 \\
\hline 5 & 0,7816 & 680 & 765 & 2209 & 2759 \\
\hline 6 & 0,8308 & & & 680 & 677 \\
\hline
\end{tabular}


Tabla 3

Perfil de la dependencia: características de los grupos

\begin{tabular}{|c|c|c|c|}
\hline Variable & Categoría & \% del grupo con categoría & \% de categoría en el grupo \\
\hline \multicolumn{4}{|c|}{ Clase I: 7.003 individuos $(34,63 \%)$} \\
\hline Sensoriales & No & 61,69 & 39,87 \\
\hline Ciego_sordo & No & 98,06 & 35,45 \\
\hline Comunicación_cognitivas & No & 93,96 & 41,34 \\
\hline Movilidad & Sin dependencia & 66,50 & 56,14 \\
\hline AVD & No & 95,82 & 42,85 \\
\hline AIVD & No & 78,87 & 49,94 \\
\hline Edad & $40-64$ & 32,33 & 40,92 \\
\hline Edad & $65-79$ & 41,11 & 36,86 \\
\hline Sexo & Varón & 43,61 & 35,69 \\
\hline \multicolumn{4}{|c|}{ Clase II: 4.747 individuos $(23,47 \%)$} \\
\hline Sensoriales & Sin dependencia & 81,84 & 55,64 \\
\hline Sensoriales & Dependencia moderada & 16,96 & 36,61 \\
\hline Ciego_sordo & No & 99,79 & 24,46 \\
\hline Comunicación_cognitivas & No & 99,28 & 29,61 \\
\hline Movilidad & No & 99,07 & 83,22 \\
\hline AVD & No & 99,47 & 30,15 \\
\hline AIVD & No & 98,59 & 42,31 \\
\hline Sexo & Varón & 50,81 & 28,18 \\
\hline Edad & $65-79$ & 42,87 & 26,06 \\
\hline \multicolumn{4}{|c|}{ Clase III: 4.234 individuos $(20,94 \%)$} \\
\hline Sensoriales & No & 86,28 & 33,71 \\
\hline Ciego_sordo & $\mathrm{Si}$ & 11,64 & 57,66 \\
\hline Comunicación_cognitivas & Sin dependencia & 36,16 & 65,46 \\
\hline Comunicación_cognitivas & Dependencia moderada & 11,34 & 46,74 \\
\hline Movilidad & Sin dependencia & 74,28 & 37,91 \\
\hline Movilidad & Dependencia moderada & 22,32 & 23,77 \\
\hline AVD & No & 82,03 & 22,18 \\
\hline AVD & Sin dependencia & 12,66 & 64,89 \\
\hline AIVD & Sin dependencia & 46,84 & 74,19 \\
\hline AIVD & Dependencia moderada & 24,52 & 56,47 \\
\hline AIVD & Dependencia grave & 7,82 & 26,87 \\
\hline Sexo & Mujer & 62,87 & 22,82 \\
\hline Edad & $6-39$ & 17,43 & 29,63 \\
\hline Edad & $40-64$ & 30,09 & 23,03 \\
\hline \multicolumn{4}{|c|}{ Clase IV: 3.475 individuos $(17,18 \%)$} \\
\hline Sensoriales & No & 66,59 & 21,36 \\
\hline Sensoriales & Dependencia moderada & 12,98 & 20,51 \\
\hline Comunicación_cognitivas & Dependencia moderada & 13,24 & 44,79 \\
\hline Comunicación_cognitivas & Dependencia grave & 12,00 & 62,43 \\
\hline Movilidad & Dependencia moderada & 47,51 & 41,52 \\
\hline Movilidad & Dependencia grave & 35,40 & 80,44 \\
\hline AVD & Dependencia moderada & 25,61 & 72,24 \\
\hline AVD & Dependencia grave & 30,16 & 93,49 \\
\hline AVD & Dependencia total & 18,16 & 45,59 \\
\hline AIVD & Dependencia moderada & 10,30 & 19,48 \\
\hline AIVD & Dependencia grave & 19,65 & 55,44 \\
\hline AIVD & Dependencia total & 60,92 & 61,88 \\
\hline Sexo & Mujer & 64,60 & 19,24 \\
\hline Edad & $>80$ & 40,46 & 32,03 \\
\hline
\end{tabular}


Tabla 3 (continuación)

Perfil de la dependencia: características de los grupos

\begin{tabular}{|l|c|c|c|}
\hline \multicolumn{1}{|c|}{ Variable } & Categoría & \% del grupo con categoría & \% de categoría en el grupo \\
\hline \multicolumn{3}{|c|}{ Clase V: 765 individuos (3,78\%) } \\
\hline Sensoriales & No & 70,85 & 5,00 \\
\hline Ciego_sordo & $\mathrm{Si}$ & 11,50 & 10,29 \\
\hline Comunicación_cognitivas & Dependencia moderada & 7,84 & 5,84 \\
\hline Comunicación_cognitivas & Dependencia grave & 27,71 & 31,74 \\
\hline Movilidad & Dependencia total & 84,84 & 83,96 \\
\hline AVD & Dependencia total & 93,46 & 51,66 \\
\hline AIVD & Dependencia total & 94,64 & 21,16 \\
\hline Edad & $>80$ & 44,58 & 7,77 \\
\hline Sexo & Mujer & 62,09 & 4,07 \\
\hline
\end{tabular}

Tabla 4

Características de la dependencia en la muestra de discapacitados

\begin{tabular}{|c|c|c|}
\hline Variable & Categoría & $\%$ del total \\
\hline \multirow{5}{*}{ Sensoriales } & No & 53,58 \\
\hline & Sin dependencia & 34,53 \\
\hline & Dependencia moderada & 10,87 \\
\hline & Dependencia grave & 0,89 \\
\hline & Dependencia total & 0,11 \\
\hline \multirow{2}{*}{ Ciego_sordo } & No & 95,77 \\
\hline & $\mathrm{Si}$ & 4,23 \\
\hline \multirow{5}{*}{ Comunicación_cognitivas } & No & 78,70 \\
\hline & Sin dependencia & 11,57 \\
\hline & Dependencia moderada & 5,08 \\
\hline & Dependencia grave & 3,30 \\
\hline & Dependencia total & 1,35 \\
\hline \multirow{5}{*}{ Movilidad } & No & 27,94 \\
\hline & Sin dependencia & 41,02 \\
\hline & Dependencia moderada & 19,66 \\
\hline & Dependencia grave & 7,56 \\
\hline & Dependencia total & 3,82 \\
\hline \multirow{5}{*}{ AVD } & No & 77,44 \\
\hline & Sin dependencia & 4,08 \\
\hline & Dependencia moderada & 6,09 \\
\hline & Dependencia grave & 5,54 \\
\hline & Dependencia total & 6,84 \\
\hline \multirow{5}{*}{ AIVD } & No & 54,69 \\
\hline & Sin dependencia & 13,22 \\
\hline & Dependencia moderada & 9,09 \\
\hline & Dependencia grave & 6,09 \\
\hline & Dependencia total & 16,92 \\
\hline \multirow{4}{*}{ Edad } & $6-39$ & 12,32 \\
\hline & $40-64$ & 27,36 \\
\hline & $65-79$ & 38,62 \\
\hline & $>80$ & 21,71 \\
\hline \multirow{2}{*}{ Sexo } & Varón & 42,32 \\
\hline & Mujer & 57,68 \\
\hline
\end{tabular}

En la tabla 4 se presentan las características del conjunto de la población discapacitada en relación a las categorías de dependencia, edad y sexo. Se observa que la discapacidad más frecuente es la de movilidad, seguida de las sensoriales y las actividades instrumentales de la vida diaria. También que los niveles de dependencia más graves son más frecuentes en los dos tipos de actividades de la vida diaria. Los valores de esta tabla permiten comparar las diferencias en la composición de cada clase y el conjunto de la población discapacitada.

\section{DISCUSIÓN}

Este estudio perfila la situación de la dependencia en España a partir de los datos disponibles sobre las discapacidades que padecen las personas y el nivel de gravedad que estas comportan. Se enmarca en un contexto muy empírico en el que los datos han sido diseñados a partir de un esquema conceptual no específico para la dependencia, pero debido a la enorme importancia que tiene conocer la gravedad y las consecuencias de la discapacidad en la vida diaria, creemos que se justifica esta aproximación oportunista mediante los datos disponibles.

Uno de los resultados más relevantes de nuestro estudio indica que determinados 
niveles de gravedad se relacionan más con unas discapacidades que con otras. Por este motivo los análisis comparativos basados en la discapacidad que no tengan en cuenta la gravedad, pueden llevar a conclusiones sesgadas, aunque actualmente no existe una única forma de ponderar esta gravedad. El sistema de puntuación de gravedad propuesto en este estudio podría ser una herramienta útil para dilucidar esta cuestión en futuros trabajos.

Un trabajo reciente ${ }^{19}$ ha puesto de manifiesto el efecto que tiene sobre la prevalencia de discapacidad el hecho de utilizar métodos de puntuación distintos. Aunque los resultados de nuestro estudio no sean estrictamente comparables (aquel trabajo se basa solo en las actividades de la vida diaria), las conclusiones de ambos son consistentes. Alegre et al utilizan un método de puntuación alternativo que presupone que las limitaciones en estas actividades son aditivas e independientes entre si. En nuestro estudio se ha computado la gravedad media para cada tipo de discapacidad, de forma que se asume que las limitaciones dentro del grupo de discapacidad están relacionadas, pero que los distintos tipos de discapacidad son independientes entre si. Este criterio se basa en los resultados que muestran que la gravedad no es independiente del tipo de discapacidad padecida y que las discapacidades específicas de un grupo se sitúan cercanas en el plano respecto de la gravedad. Aunque todos los sistemas de ponderación de la gravedad comportan una cierta arbitrariedad, parece más coherente utilizar esta puntuación media obtenida para cada tipo de discapacidad de forma independiente, aunque probablemente este criterio sea más conservador que el usado por los otros autores ${ }^{19,20}$. Por otra parte, la agrupación en cinco tipos distintos de discapacidad que se ha usado en este trabajo tampoco ha sido escogida de manera arbitraria. Un análisis realizado previa- mente $^{21}$ mostraba que la agrupación alternativa obtenida mediante métodos estadísticos no ofrecían muchas ventajas respecto al uso de un criterio de agrupación funcional, que ya ha sido utilizado anteriormente por otros autores ${ }^{15-16,20} \mathrm{y}$ es el que finalmente se adoptó.

Otra aportación relevante de este trabajo es la descripción del perfil de la dependencia en la población española, que se caracteriza en cinco grupos, con unos niveles de gravedad ascendentes. En los de menor dependencia se sitúan predominantemente las personas jóvenes y de edades medias, los varones y las discapacidades sensoriales, mientras que en los dependencia elevada o total las mujeres, los mayores de 80 años y las discapacidad de movilidad y en las actividades de la vida diaria. Estos resultados son coherentes con los de otros estudios $5-6,19,22-23$, aunque la mayoría de ellos se basan únicamente en las dificultades para las actividades diarias. Sin embargo, nuestro trabajo muestra que una proporción importante de la población discapacitada, ve agravada su dependencia en las actividades básicas por la coexistencia de otras discapacidades (cognitivas, sensoriales o de movilidad) y pone de manifiesto la importancia de tener en cuenta el conjunto de las discapacidades que padece el individuo y no tan solo aquellas que afectan las actividades diarias.

Las características de los distintos grupos de población dependiente identificadas en este estudio podrían ser tenidas en cuenta a la hora de definir la población vulnerable o susceptible de distintos grados de intervención, desde preventivas a asistenciales ${ }^{13}$. Además, los resultados presentados permiten, estimar de manera general los efectivos de población que se encuentran en cada situación y extrapolarlos a la población general. Este hecho puede ser de gran ayuda de cara a estimar los recursos necesarios en 
ayudas económicas y de servicios sociales o de salud, en el contexto de la aplicación de la nueva ley de dependencia ${ }^{14}$. También, la posibilidad de diferenciar distintos grados de discapacidad en base a su gravedad, comorbilidad o niveles de limitación funcional, permitiría ajustar muchos indicadores que se derivan de estos datos, como por ejemplo los cálculos de la esperanza de vida libre de discapacidad $^{24,25}$.

Una de las limitaciones del estudio, consecuencia de la utilización oportunista de los datos disponibles, es la medida del componente de discapacidad y su gravedad, complicada por el hecho de partir de una lista de 36 discapacidades, con distinto nivel de gravedad y correlación entre si. La importancia de profundizar en los conceptos que se manejan reside en el hecho de que, en función del modelo conceptual, los instrumentos de medida de la discapacidad y la dependencia pueden ser distintos y en consecuencia la estimación de su frecuencia y distribución a nivel poblacional puede ser diferente ${ }^{26}$.

La encuesta de la que se han obtenido los datos, se basa en el modelo de discapacidad de la $\mathrm{OMS}^{27}$ en el cual las diferentes dimensiones de la salud y la discapacidad están relacionadas entre sí como distintos eslabones de una cadena y se asume una secuencia temporal que une cada una de ellas. Sin embargo muchas evidencias muestran que estas relaciones no son unidireccionales, al contrario, pueden actuar en diferentes sentidos (empeorar, recuperar la funcionalidad, la muerte, etc) ${ }^{12}$ Además, los datos proceden de un diseño transversal y por ello aunque el modelo conceptualmente lo permitiera, la multidireccionalidad entre las variables no puede ser tenida en cuenta a efectos del análisis. En el ámbito clínico es habitual trabajar con escalas de medida para evaluar la capacidad funcional y la dependencia que han sido diseñadas ad-hoc y vali- dadas previamente en el contexto asistencial. Sin embargo, este procedimiento de diseño y validación experimental de escalas de medida para la obtención de indicadores de referencia del conjunto poblacional no es tan frecuente. En este sentido los trabajos con la National Long-Term Care Survey de Estados Unidos ${ }^{2}$ y otras experiencias más recientes ${ }^{26}$ pueden constituir una referencia útil y contrastada de clasificación funcional de las discapacidades para su utilización en estudios poblacionales.

En conclusión, el tipo de discapacidad se asocia a su gravedad y esto comporta distintos grados de dependencia. Se identifican cinco grupos de personas dependientes con niveles de dependencia ascendente. Los primeros con dependencia leve o moderada se asocian a varones y de edades medias y los de mayor dependencia se relacionan con el sexo femenino y las edades más elevadas. Este estudio permite estimar su frecuencia global dentro de la población, lo que puede ser de gran utilidad para estimar los recursos necesarios para la atención sanitaria y social que precisen.

\section{AGRADECIMIENTOS}

A M a Arán Barés, Jaume March, Anna Puigdefàbregas, $\mathrm{M}^{\mathrm{a}}$ Mar Torné y Roser Martí, por su colaboración en diferentes fases del proyecto y a Francisco Viciana, por los comentarios sucesivos. Agradecemos también los comentarios de las personas que han hecho la evaluación anónima, la cual ha permitido mejorar este original.

\section{BIBLIOGRAFÍA}

1. Ignasi Serra, Rosa Gispert, Xavier Puig, $\mathbf{M}^{\mathrm{a}}$ del Mar Torné, Anna Puigdefàbregas. Impacte de l'edat I les causes de mort en els canvis de l'esperança de vida. Catalunya, 1987-2002. Barcelona, Departament de Salut. Generalitat de Catalunya 2006. Disponible 
en: http://www.gencat.net/salut/depsan/units/sanitat/html/ca/xifres/index.html

2. Manton KG, Corder L, Stallard E. Chronic disability trends in elderly United States populations: 1982-1994 Proc. Natl. Acad. Sci. USA.1997;vol 94. Medical Sciences.

3. Graciani A, Banegas JR, López-García, Rodriguez-Artalejo F. Prevalence of disability and associated social and health-related factors among the elderly in Spain: a population-based study. Maturitas 2004;48:381-92.

4. Beland F, Zunzunegui MV. Predictors of functional status in older people living at home. Age Ageing 1999;28:153-9.

5. Garcés J, Ródenas F, Sanjosé V. Care needs among the dependent population in Spain: an empirical approach. Health Soc Care Community 2004; 12(6):466-474.

6. Otero A, Zunzunegui MV, Rodriguez-Laso A, Aguilar D, Lázaro P. Volumen y tendencias de la dependencia asociada al envejecimiento en la población española. Rev Esp Salud Publica 2004;78:201-13.

7. Mackenbach JP, Borsboom GJJM, Nusselder WJ, Looman CWN, Schrijvers CTM. Determinants of levels and changes of physical functioning in Chronically ill persons: results from the GLOBE study. J Epidemiol Community Health 2001;55:631-8.

8. Matthews R, Smith L, Hancock R, Jagger C, Spiers N. Socioeconomic factors associated with the onset of disability in older age: a longitudinal study of people aged 75 years and over. Soc Science Med. 2005;61:1567-75.

9. Valderrama-Gama E, Damián J, Ruigómez A, Martín-Moreno JM. Chronic disease, functional status and self-ascribed causes of disabilities among noninstitutionalized older people in Spain. J Gerontol A Biol Sci Med Sci. 2002 Nov; 57(11): M716-21.

10. Consejo de Europa. Recomendación núm. R (98): 9, del Consejo de ministros a los estados miembros relativo a la dependencia. Luxemburgo: Oficina de Publicaciones Oficiales de las Comunidades Europeas; 1998.

11. Ministerio de Trabajo y Asuntos Sociales, Imserso. La Atención a las personas en situación de dependencia en España. Libro Blanco. Madrid: MTAS, IMSERSO; 2004. Disponible en: http://www.mtas.es//inicioas/dependencia/depen.htm
12. Zunzunegui MV, Nuñez O, Durban M, García de Yebenes MJ, Otero A. Decreasing prevalence of disabilitie of daily living, functional limitation and poor self-rated health: a 6 year follow-up study in Spain. Aging Clin Exp Res. 2006;18(5):352-8.

13. Fundació Institut Català de l'Envelliment, UAB. Estudio del modelo de atención a las personas mayores con dependencia en España. Barcelona: FICE; 2004.

14. Ministerio de trabajo y asuntos sociales. Ley 39/2006 de 14 de Diciembre de promoción de la autonomía personal y atención a las personas en situación de dependencia.BOE 299 núm de 15/12/2006.

15. Instituto Nacional de Estadística. Encuesta sobre discapacidades, deficiencias y estado de salud. 1999. Madrid: INE; 2000. Disponible en: http://www.imsersomayores.csic.es/documentos/b usquedas/registro.jsp?idDoc $=1058$

16. Puga Gonzalez, Ma. D., Abellán Garcia, A. El proceso de discapacidad. Un análisis de la Encuesta sobre discapacidades, deficiencias y estado de salud. Alcobendas: Fundación Pfizer;2004.

17. Ward JH. Hierarchical grouping to optimize an objective function. J Am Stat Assoc. 1963, 77, 841-847.

18. Lebart L, Morineau A. SPAD, Système Portable pour l'Analyse des Données. Paris: CISIA ; 1972.

19. Alegre Escolan A, Ayuso Gutierrez M, Guillén Estany M, Monteverde Verdenelli M, Pociello García E. Tasa de dependencia de la población española no institucionalizada y criterios de valoración de la severidad. Rev Esp Salud Pública 2005;79:351-63.

20. Viciana Fernández F, Hernández Rodríguez JA, Canto Casasola VD, Avila Garzón AI. Longevidad y Calidad de Vida en Andalucía. Sevilla: IEA; 2003. Disponible en: www.juntadeandalucia.es/institutodeestadistica/lcva/lcva.pdf

21. Clot-Razquin G, Gispert R, Freitas A, March J, Torné MM. El perfil de la discapacidad en España a partir de la encuesta de 1999. Gac Sanit 2007;21(Espec Congr):40.

22. Albarrán Lozano I, Alonso Gonzalez P. Clasificación de las personas dependientes a partir de la encuesta de discapacidades, deficiencias y estado de salud de 1999. Rev Esp Salud Pública 2006;80:349-60. 
23. Cabero Gracia J. Indicadores de discapacidad en la encuesta de discapacidades, deficiencias y estado de salud. Rev Esp Salud Pública 2007; 81(2):167-181

24. Gispert R, Ruíz-Ramos M, Arán Bares Maa Viciana F, Clot-Razquin G. Diferencias en la esperanza de vida libre de discapacidad por sexo y Comunidades Autónomas en España. Rev Esp Salud Pública 2007; 81(2):155-165.

25. Ruiz-Ramos M, Viciana-Fernandez F. Desigualdades en longevidad y calidad de vida entre Andalucía y España. Gac Sanit. 2004;18(4):260-7.
26. Pluijm SMF, Bardage C, Nikula S, Blumstein T, Jylha M, Minicuci N, Zunzunegui V, Pedersen NL, Deeg D. A harmonized measure of activities of daily living was a reliable and valid instrument for comparing disability in older people across countries. J Clin Epidemiol. 2005; 58:1015-23.

27. World Health Organization. International Classification of Functioning, Disability and Health. 2nd ed. Geneva: WHO; 2001. 
